\title{
BERNARD LACOLLE
}

Maximisation globale de la norme euclidienne sur un compact : résolution approchée par utilisation des problèmes projetés

RAIRO - Analyse numérique, tome 13, nº 2 (1979), p. 101-117.

$<$ http://www.numdam.org/item?id=M2AN_1979_13_2_101_0>

(C) AFCET, 1979, tous droits réservés.

L'accès aux archives de la revue «RAIRO - Analyse numérique » implique l'accord avec les conditions générales d'utilisation (http://www.numdam.org/ legal.php). Toute utilisation commerciale ou impression systématique est constitutive d'une infraction pénale. Toute copie ou impression de ce fichier doit contenir la présente mention de copyright.

\section{Numdam}




\title{
MAXIMISATION GLOBALE \\ DE LA NORME EUCLIDIENNE SUR UN COMPACT : RÉSOLUTION APPROCHÉE PAR UTILISATION DES PROBLÈMES PROJETÉS $\left({ }^{*}\right)$
}

\author{
par Bernard LACOLle $\left({ }^{1}\right)$ \\ Communiqué par F ROBERT
}

\begin{abstract}
Résumé. - Dans cet article, nous décrivons une méthode de maxımısatıon globale de la norme euchdienne usuelle sur un compact $\mathscr{D}$ de $\mathbf{R}^{n}$ lorsque $n$ est petit La méthode demande uniquement la maximisatıon de fonctionnelles linéaires sur $\mathscr{D}$ mals aucune hypothèse supplémentaire sur $\mathscr{D}$ n'est nécessatre
\end{abstract}

Abstract - In this paper, we describe a method for global maximisation of the usual euclidian norm subject to a compact domain $\mathscr{D}$ of $\mathbf{R}^{n}$ when $n$ is small The method only requires maximisation of linear functionals on $\mathscr{D}$ but no supplementary hypothesis about $\mathscr{D}$ is needed

\section{INTRODUCTION}

Soient $\mathscr{D}$ une partie compacte de $\mathbf{R}^{p}$ et $\varphi_{2}$ la norme euclidienne usuelle sur $\mathbf{R}^{p}$. Le problème consistant à calculer $X^{*} \in \mathscr{D}$ tel que $\varphi_{2}\left(X^{*}\right)=\underset{X \in \mathscr{D}}{\operatorname{Max}} \varphi_{2}(X)$ ne peut être abordé actuellement que dans des cas extrêmement élémentaires. Deux raisons essentielles à cela : la première est que, même lorsque $\mathscr{D}$ est « régulier » (convexe par exemple) l'existence très fréquente de maximums locaux de la norme $\varphi_{2}$ sur $\mathscr{D}$ (illustrée sur la figure 1) écarte en général toutes les méthodes d'optimisation classiques qui utilisent une croissance locale de la fonction à maximiser; ces méthodes convergent en effet vers un maxımum local. La seconde raison est que la plupart des algorithmes actuellement existant de maximisation globale [1] ne sont pratiquement utilisables que si la dimension de l'espace est inférieure ou égale à 2 .

Les exemples à l'origine de ce travail nous ont suggéré l'utilisation de problèmes annexes au problème initial consistant à trouver pour $u \in \mathbf{R}^{p}$ un vecteur $X_{u} \in \mathscr{D}$ tel que

$$
X_{u}^{T} u=\underset{X \in \mathscr{D}}{\operatorname{Max}} X^{T} u
$$

(*) Reçu mar 1978

(1) Unıversité scientıfique et médıcale, Laboratoıre de Mathématıques applıquěes, Grenoble.

R A I R O Analyse numérique/Numerical Analysis, 0399-0516/1979/101/\$ 400

(C) Bordas-Dunod 


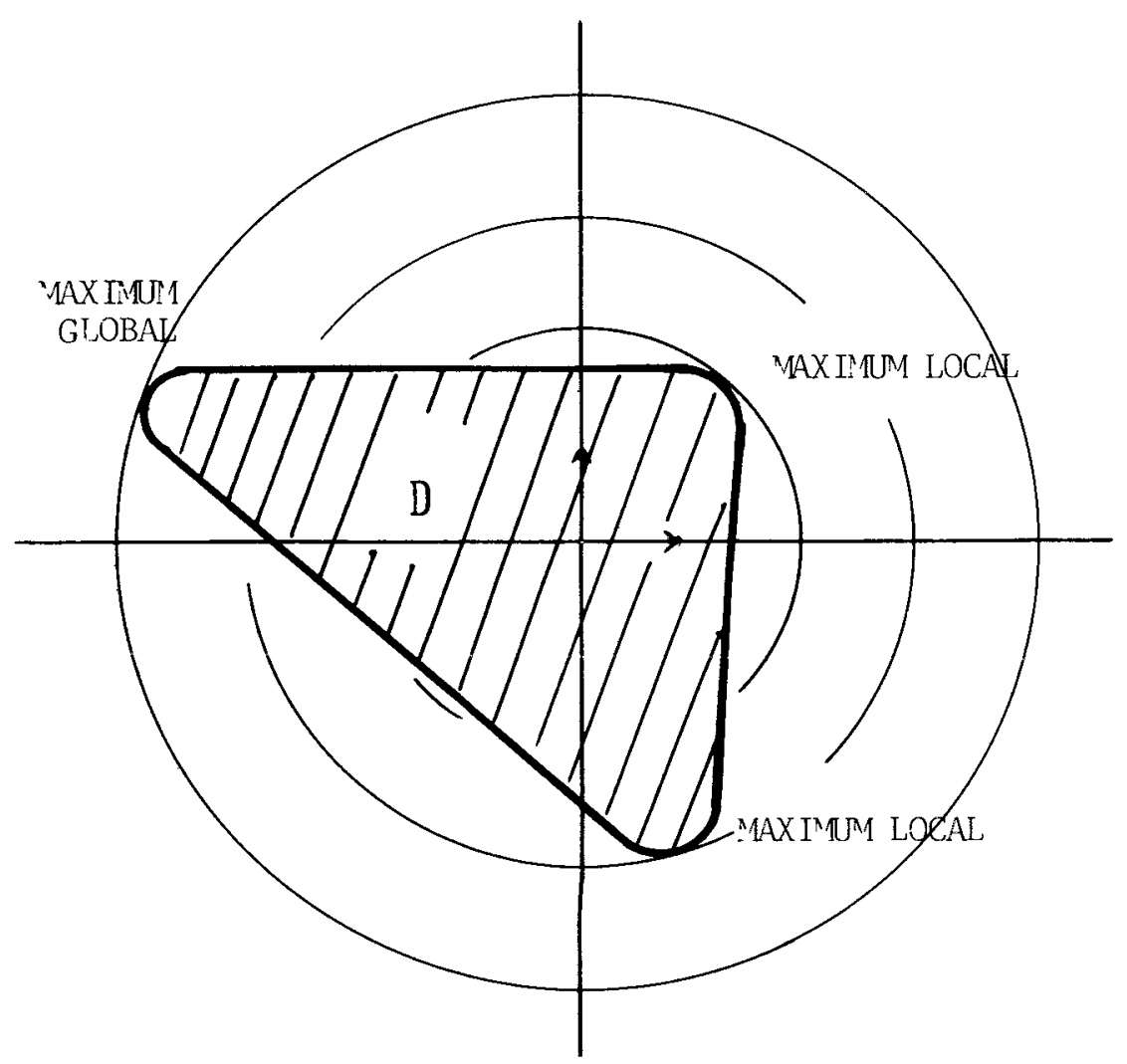

Figure 1

Ces problèmes de maximisation de fonctionnelles linéaires sur $\mathscr{D}$ (illustrés dans $\mathbf{R}^{2}$ par la figure 2) constitueront l'outil de base de notre méthode. La seule hypothèse faite sur le compact $\mathscr{D}$ sera que l'on puisse, à $u$ quelconque de $\mathbf{R}^{p}$, associer une solution $X_{u}$ du problème "projeté » sur $u$.

D'autre part, la recherche d'une solution aux précisions habituellement utilisées étant en général impossible, nous chercherons à obtenir une solution maximisant la norme $\varphi_{2}$ à une précision relative donnée relativement grossière (de l'ordre de 5 à $10 \%$ ou même plus).

L'essentiel de ce travail consiste à montrer comment on peut choisir uniquement en fonction de $p$ et de la précision demandée, et donc indépendamment de $\mathscr{D}$ un certain nombre de directions de projections de façon à déduire très simplement à partir des solutions des problèmes "projetés ", associés aux directions précédentes, une solution du problème initial à la précision demandée. 


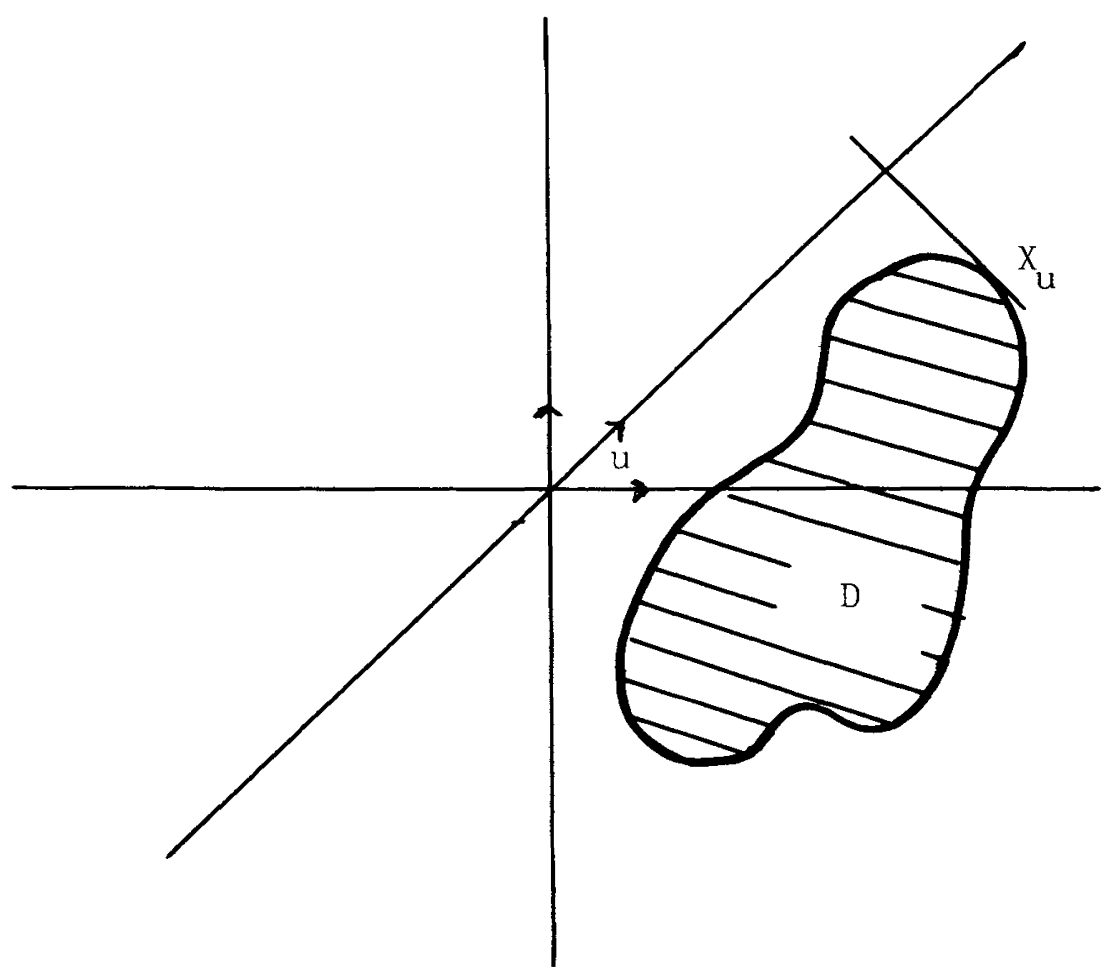

Figure 2

Cette méthode nous permettra d'aborder des problèmes dans un espace de dimension 4 ou 5 avec des temps de calcul fort raisonnables.

Nous montrerons dans la section 1 un exemple de problème ayant motivé ce travail. L'algorithme utilisé sera décrit et étudié dans la section 2. D'autres exemples seront donnés dans la section 3 .

\section{NOTATIONS ET DÉFINITIONS PRELIMINAIRES}

$V$ (i) désignera la $i$-ième coordonnée du vecteur $V$ de $\mathbf{R}^{p} . \mathscr{M}_{n, n}$ désignera l'ensemble des matrices à $n$ lignes et $n$ colonnes à coefficients réels, $A(i, j)$ désignera le terme de la $i$-ième ligne $j$-ième colonne de la matrice $A$.

Nous appellerons problème de distance euclidienne maximale (associé à $\mathscr{D}$ compact de $\mathbf{R}^{p}$ ) le problème consistant à trouver $X^{*} \in \mathscr{D}$ tel que :

$$
\varphi_{2}\left(X^{*}\right)=\operatorname{Max}_{X \in \mathscr{D}} \varphi_{2}(X) .
$$


Nous appellerons problème projeté (sur la direction $u$ de $\mathbf{R}^{p}$ ) le problème consistant à trouver $X_{u} \in \mathscr{D}$ tel que

$$
X_{u}^{T} u=\underset{X \in \mathcal{M}}{\operatorname{Max}} X^{T} u .
$$

\section{EXEMPLE INTRODUCTIF}

Le problème que nous allons décrire ici est une version très simplifiée d'un problème combinatoire (non résolu) intervenant dans l'étude de la restructuration optimale de programmes se déroulant dans des systèmes à pagination [3].

Nous montrerons comment formaliser ce problème en un problème de distance euclidienne maximale et la manière de résoudre les problèmes projetés : ceci placera le problème dans le cadre de la méthode exposée au paragraphe 2 et nous donnerons les résultats d'expériences numériques au paragraphe 2.5.

\subsection{Un problème de partitionnement [4]}

Soit $E=\{1,2, \ldots, 2 n\}, n \in \mathbf{N}^{*}$ ( $n$ de l'ordre de 50) et $A$ une matrice de $\mathscr{M}_{2 n, 2 n}(\mathbf{R})$ donnée par

$$
A=\sum_{\imath=1}^{p} B_{\imath} B_{\imath}^{T} \quad \text { où } \quad B_{\imath} \in \mathbf{R}^{2 n}, \quad i=1,2, \ldots, p
$$

et $p$ est faible (de l'ordre de 5).

Le probleme est de partitionner $t$ en deux sous-ensembles $E_{1}$ et $E_{2}$ comportant chacun $n$ éléments et tels que : $\sum_{\substack{i \in E_{1} \\ l \in E_{2}}} A(l, j)$ soit mınımum.

Signalons la complexité du problème : dans le cas où $A$ est une matrice symétrique à coefficients dans $\{0,1\}$ le problème combinatorre est $N-P$ complet [2].

1.2. Formulation en terme de problème de « distance euclidienne maximale »

La partition de $E$ peut être caractérisée par un élément de $\mathscr{K}$ :

$$
\mathscr{K}=\left\{K \in \mathbf{R}^{2 n}: K(i)= \pm 1, i \in E, \sum_{i \in E} K(i)=0\right\}
$$

avec

$$
E_{1}=\{\imath \in E: K(i)=+1\}, \quad E_{2}=\{i \in E: K(i)=-1\} .
$$


Compte tenu que

$$
\sum_{\substack{l \in E_{1} \\ j \in E_{2}}} A(l, j)=\frac{1}{4}\left[\sum_{\substack{i \in E \\ j \in E}} A(i, j)-K^{T} A K\right] .
$$

Le problème est donc de trouver $K^{*} \in \mathscr{K}$ tel que

$$
K^{* T} A K^{*}=\underset{K \in \mathscr{K}}{\operatorname{Max}} K^{T} A K .
$$

Or

$$
K^{T} A K=K^{T}\left(\sum_{\imath=1}^{p} B_{\imath} B_{\imath}^{T}\right) K=\sum_{\imath=1}^{p}\left(K^{T} B_{\imath}\right)^{2}
$$

Posons

$$
\mathscr{D}=\left\{X \in \mathbf{R}^{p}: X(i)=K^{T} B_{\imath}, i=1,2, \ldots, p, K \in \mathscr{K}\right\} .
$$

Le problème initial peut donc se formuler ainsi :

Trouver $X^{*} \in \mathscr{D}$ et $K^{*} \in \mathscr{K}$ tels que

$$
\begin{gathered}
\varphi_{2}\left(X^{*}\right)=\operatorname{Max}_{X \in \mathscr{D}} \varphi_{2}(X), \\
K^{* T} B_{\imath}=X^{*}(i), \quad i=1,2, \ldots, p .
\end{gathered}
$$

Notons que $\mathscr{D}$ est symétrique par rapport à l'origine.

\section{3. «Problèmes projetés "}

Lemme : Soit $B \in \mathbf{R}^{2 n} . K_{B} \in \mathscr{K}$ vérifie $K_{B}^{T} B=\operatorname{Max} K^{T} B$ si et seulement si

$$
\left.\begin{array}{l}
K_{B}(i)=+1 \\
K_{B}(j)=-1
\end{array}\right\} \Rightarrow B(i) \geqq B(J) .
$$

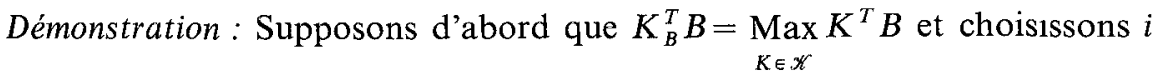
et $j$ tels que $K_{B}(i)=1, K_{B}(j)=-1$.

Considérons un vecteur $K$ ne différant de $K_{B}$ que par

$$
K(i)=-1 \quad \text { et } \quad K(j)=+1 .
$$

Nous avons

$$
K_{B}^{T} B-K^{T} B=2(B(i)-B(J)) \geqq 0 .
$$


Réciproquement, considérons un vecteur $K_{B}$ verifiant

$$
\left.\begin{array}{l}
K_{B}(l)=+1 \\
K_{B}(J)=-1
\end{array}\right\} \Rightarrow B(l) \geqq B(J)
$$

et $K$ un vecteur quelconque de $\mathscr{K}$ Posons

$$
\begin{gathered}
E_{+}^{+}=\left\{l \in E \quad K(l)=K_{B}(l)=1\right\}, \\
E_{+}^{-}=\left\{l \in E \quad K(l)=+1, K_{B}(l)=-1\right\}, \\
E_{-}^{+}=\left\{l \in E \quad K(l)=-1, K_{B}(l)=1\right\}, \\
E_{-}^{-}=\left\{l \in E \quad K(l)=K_{B}(l)=-1\right\}
\end{gathered}
$$

Nous avons

$$
K_{B}^{T} B-K^{T} B=2\left(\sum_{i \in E^{+}} B(l)-\sum_{\imath \in E_{+}} B(\imath)\right)
$$

De plus

$$
n=\operatorname{Card}\left(E_{+}^{+}\right)+\operatorname{Card}\left(E_{-}^{+}\right), \quad n=\operatorname{Card}\left(E_{+}^{+}\right)+\operatorname{Card}\left(E_{+}^{-}\right)
$$

Donc

$$
\operatorname{Card}\left(E_{-}^{+}\right)=\operatorname{Card}\left(E_{+}^{-}\right)
$$

Or si $l \in E_{-}^{+}$et $\jmath \in E_{\mp}^{-}$alors $B(l) \geqq B(\jmath)$ ll en résulte donc que $K_{B}^{T} B \geqq K^{T} B$

Propriete Solt $u \in \mathbf{R}^{p}$ On peut trouver $X u \in \mathscr{D}$ et $K u \in \mathscr{K}$ verifiant

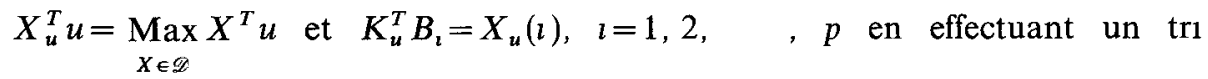
des composantes du vecteur $\sum_{\imath=1}^{p} u(\imath) B_{\imath}$

Démonstration

$$
X^{T} u=\sum_{\imath=1}^{p}\left(K^{T} B_{\imath}\right) u(\imath)=K^{T}\left(\sum_{\imath=1}^{p} u(\imath) B_{\imath}\right)
$$

s1

$$
X(l)=K^{T} B_{\imath}, \quad l=1,2, \quad, p
$$

En posant $B=\sum_{\imath=1}^{p} u(l) B_{\imath}$ et en appliquant le lemme précédant nous pouvons trouver un vecteur $K_{u}$ tel que $K_{u}^{T} B=\underset{K \in \mathscr{X}}{\operatorname{Max}} K^{T} B$ et le vecteur $X_{u}$ correspondant en ordonnant par ordre décroissant les composantes de $B$ 


\section{ALGORITHMES ÉNUMÉRATIFS POUR LA RÉSOLUTION A PRÉCISION RELATIVE} DONNÉE DE PROBLĖMES DE DISTANCE EUCLIDIENNE MAXIMALE

\section{1. Notations}

$\mathscr{U}(p)=\left\{u \in \mathbf{R}^{p}: \varphi_{2}(u)=+1\right\}$,

$\mathscr{U}^{+}(p)=\{u \in \mathscr{U}(p): u(i) \geqq 0, i=1,2, \ldots, p\}$,

$\mathscr{D}$ est un compact de $\mathbf{R}^{p}$ non réduit à $\{0\}$.

Problème de « DISTANCE euclidienne MAXIMALE »: Nous nous proposons de trouver une solution « approchée " du problème $\operatorname{Max} \varphi_{2}(X) . X^{*} \in \mathscr{D}$ désignera dans la suite une solution exacte de ce problème.

Hypothèse : Problèmes projetés: Pour $u \in \mathbf{R}^{p}$ nous supposons savoir trouver $X_{u} \in \mathscr{D}$ tel que

$$
X_{u}^{T} u=\underset{X \in \mathscr{D}}{\operatorname{Max}} X^{T} u
$$

$X_{u}$ sera solution du problème projeté (sur $\left.u\right)$.

DÉfINITION : Soit $\varepsilon>0 . X_{\varepsilon} \in \mathscr{D}$ sera appelée solution à la précision relative $\varepsilon$ si

$$
\varphi_{2}\left(X_{\varepsilon}\right) \neq 0, \quad \frac{\varphi_{2}\left(X^{*}\right)-\varphi_{2}\left(X_{\varepsilon}\right)}{\varphi_{2}\left(X_{\varepsilon}\right)} \leqq \varepsilon .
$$

2. 2. Ensembles $\mathscr{U}_{\varepsilon}(p), \mathscr{U}_{\varepsilon}^{s}(p), \mathscr{U}_{\varepsilon}^{+}(p)$

DÉfINITION : $\mathscr{U}_{\varepsilon}(p)$ désigne un sous-ensemble de $\mathscr{U}(p)$ vérifiant :

$$
\forall u \in \mathscr{U}(p), \quad \exists u_{\varepsilon} \in \mathscr{U}_{\varepsilon}(p), \quad u_{\varepsilon}^{T} u \geqq \frac{1}{1+\varepsilon},
$$

$\mathscr{U}_{\varepsilon}^{s}(p)$ désigne un sous-ensemble de $\mathscr{U}(p)$ tel que $\mathscr{U}_{\varepsilon}^{s}(p) \cup\left(\mathscr{U}_{\varepsilon}^{s}(p)\right)$ soit un ensemble $\mathscr{U}_{\varepsilon}(p), \mathscr{U}_{\varepsilon}^{+}(p)$ désigne un sous-ensemble de $\mathscr{U}^{+}(p)$ tel que

$$
\forall u \in \mathscr{U}^{+}(p), \quad \exists u_{\varepsilon}^{+} \in \mathscr{U}_{\varepsilon}^{+}(p), \quad u_{\varepsilon}^{+T} u \geqq \frac{1}{1+\varepsilon} .
$$

Exemple : Dans $\mathbf{R}^{2}$ soient

$$
u_{1}=\left(\begin{array}{l}
1 \\
0
\end{array}\right)=-u_{3} \quad \text { et } \quad u_{2}=\left(\begin{array}{l}
0 \\
1
\end{array}\right)=-u_{4} .
$$

Pour tout vecteur $u$ de $\mathscr{U}(2)$ il existe $u_{\imath}$ tel que

$$
u_{\imath}^{T} u=\cos \theta \quad \text { avec } \quad 0 \leqq \theta \leqq \frac{\pi}{4} \quad \text { donc } u_{\imath}^{r} u \geqq \frac{1}{\sqrt{2}} .
$$




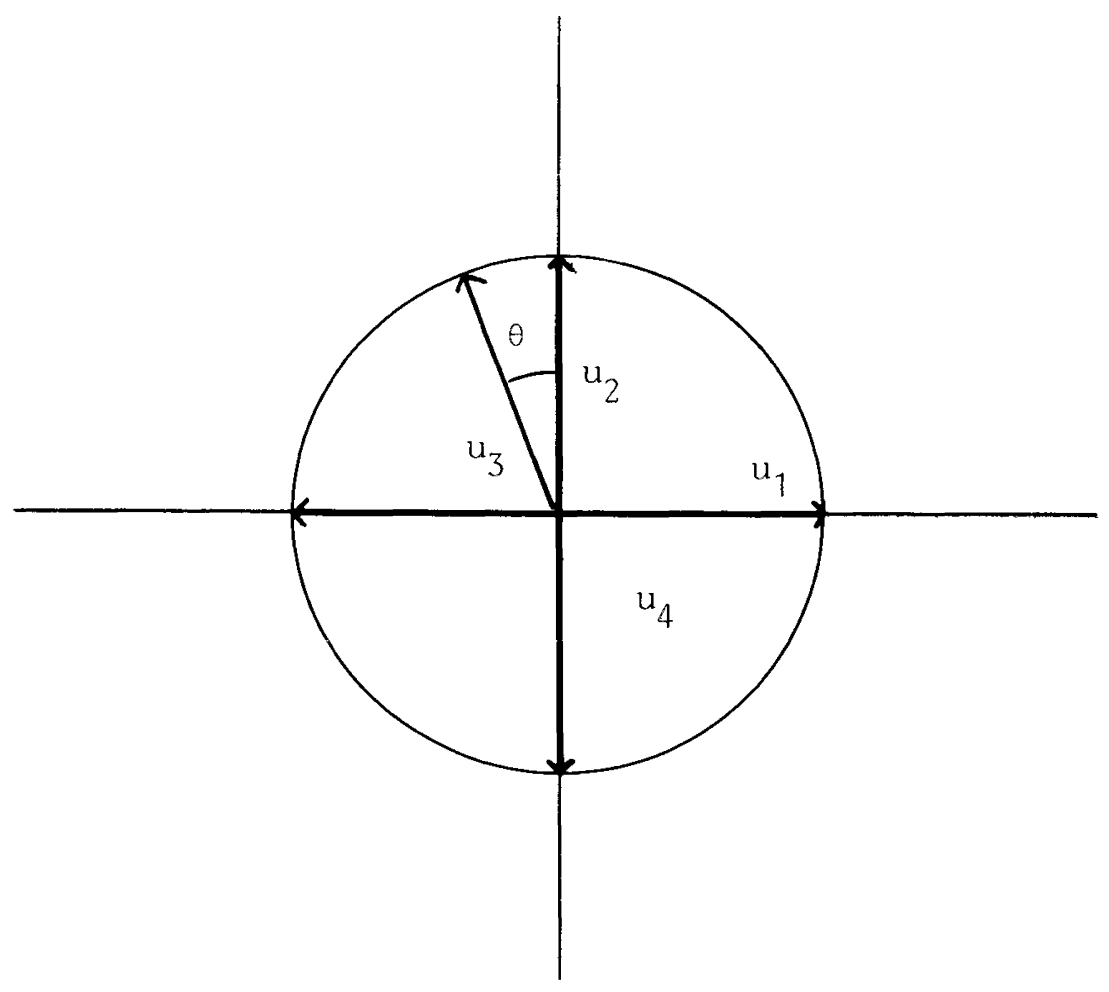

Figure 3

Nous pouvons donc poser $\mathscr{U}_{\varepsilon}(2)=\left\{u_{1}, u_{2}, u_{3}, u_{4}\right\}$ avec $\varepsilon=\sqrt{2}-1$.

Proposimion : Pour tout $\varepsilon>0$ il existe des ensembles $\mathscr{U}_{\varepsilon}(p), \mathscr{U}_{\varepsilon}^{s}(p)$ et $\mathscr{U}_{\varepsilon}^{+}(p)$ comportant un nombre fini d'éléments.

Bien qu'une démonstration directe puisse être faite, nous renvoyons au corollaire de la méthode de construction des ensembles $\mathscr{U}_{\varepsilon}(p)$ et $\mathscr{U}_{\varepsilon}^{s}(p)$ au paragraphe 2.4.

Dans toute la suite les ensembles $\mathscr{U}_{\varepsilon}(p), \mathscr{U}_{\varepsilon}^{s}(p)$ et $\mathscr{U}_{\varepsilon}^{+}(p)$ seront finis.

\subsection{Résolution à la précision relative $\varepsilon$}

Proposition : Soient $X_{\varepsilon}^{*} \in \mathscr{D}$ et $u_{\varepsilon}^{*} \in \mathscr{U}_{\varepsilon}(p)$ vérifiant :

$$
X_{\varepsilon}^{* T} u_{\varepsilon}^{*}=\operatorname{Max}_{u \in \mathscr{H}_{\varepsilon}(p)} \operatorname{Max}_{X \in \mathscr{D}} X^{T} u,
$$

$X_{\varepsilon}^{*}$ est solution à la précision relative $\varepsilon$ du problème. 
Demonstration Soit $X^{*} \in \mathscr{D}$ verifiant $\varphi_{2}\left(X^{*}\right)=\underset{X \in \mathscr{D}}{\operatorname{Max}} \varphi_{2}(X)$ et posons

$$
u^{*}=\frac{X^{*}}{\varphi_{2}\left(X^{*}\right)}
$$

Il existe $u_{\varepsilon} \in \mathscr{U}_{\varepsilon}(p)$ tel que

$$
u^{* T} u_{\varepsilon} \geqq \frac{1}{1+\varepsilon}
$$

donc

$$
\frac{\varphi_{2}\left(X^{*}\right)}{1+\varepsilon} \leqq X^{* T} u_{\varepsilon} \leqq \operatorname{Max}_{X \in \mathscr{D}} X^{T} u_{\varepsilon} \leqq X_{\varepsilon}^{* T} u_{\varepsilon}^{*}
$$

et en conséquence

$$
\frac{\varphi_{2}\left(X^{*}\right)}{1+\varepsilon} \leqq \varphi_{2}\left(X_{\varepsilon}^{*}\right)
$$

Algorithme L'algorithme consistera à résoudre tous les problèmes $\operatorname{Max} X^{T} u=X_{u}^{T} u$ pour $u \in \mathscr{U}_{\varepsilon}(p)$ et à retenir la solution maximisant $X_{u}^{T} u$ $X \in \mathscr{D}$

L'enumération sera rendue possıble sı $\mathscr{U}_{\varepsilon}(p)$ comporte un nombre d'éléments raisonnable comme ce sera le cas dans les applicatıons pratıques exposées

Proposition (Cas symetrique) $S_{l} \mathscr{D}$ est symétrique par rapport à l'orıgıne et $s l$ $X_{\varepsilon}^{*} \in \mathscr{D}$ et $u_{\varepsilon}^{*} \in \mathbb{U}_{\varepsilon}^{s}(p)$ verifient

$$
X_{\varepsilon}^{* T} u_{\varepsilon}^{*}=\underset{u \in u_{\varepsilon}(p)}{\operatorname{Max}} \underset{\lambda \in T^{T}}{\operatorname{Max}} X^{T} u
$$

alors $X_{\varepsilon}^{*}$ est solution a la precision relative $\varepsilon d u$ probleme

Démonstratıon Elle est identıque à celle de la propositıon 23 On choisit seulement $-u^{*}$ et $-u_{\varepsilon}$ si c'est $-u_{\varepsilon}$ et non $u_{\varepsilon}$ qui appartient à $\mathscr{U}_{\varepsilon}^{s}(p)$

Un algorıthme identique à l'algorithme du paragraphe 23 s'en déduit pour le cas symétrique

\subsection{Construction d'ensembles $\mathscr{U}_{\varepsilon}(p)$ et $\mathscr{U}_{\varepsilon}^{s}(p)$}

Cas $p=1 \quad \mathrm{~S}_{1} p=1, \mathscr{U}(1)$ se réduit aux deux vecteurs (1) et $(-1)$ Nous prendrons donc $\mathscr{U}_{\varepsilon}(1)=\{(1),(-1)\}$ et $\mathscr{U}_{\varepsilon}^{s}(1)=\{(1)\}$

Constructıon de $\mathscr{U}_{\varepsilon}(2)$ Divisons l'intervalle $[0,2 \pi]$ en $n$ intervalles égaux et posons

$$
\theta_{\imath}=\frac{2 \pi}{n} \quad l, \quad l=0,1, \quad, n-1
$$


On vérifie assément que si $n \geqq \pi /[\operatorname{Arccos}(1 /(1+\varepsilon))]$ nous pouvons poser

$$
\mathscr{U}_{\varepsilon}(2)=\left\{\left(\begin{array}{c}
\cos \theta_{\imath} \\
\sin \theta_{\imath}
\end{array}\right), i=0,1, \ldots, n-1\right\} .
$$

Construction de $\mathscr{U}_{\varepsilon}^{s}(2)$ : Divisons l'intervalle $[0, \pi]$ en $n$ intervalles égaux et posons

$$
\theta_{i}=\frac{\pi}{n} . i, \quad i=0,1, \ldots, n-1
$$

On vérifie aisément que si $n \geqq \pi /[2 \operatorname{Arc} \cos (1 /(1+\varepsilon))]$ nous pouvons poser

$$
\mathscr{U}_{\varepsilon}^{s}(2)=\left\{\left(\begin{array}{c}
\cos \theta_{\imath} \\
\sin \theta_{\imath}
\end{array}\right), i=0,1, \ldots, n-1\right\} \text {. }
$$

Construction de $\mathscr{U}_{\varepsilon}^{+}(2)$ : Nous nous servirons par la suite de cet ensemble. Divisons l'intervalle $[0, \pi / 2]$ en $n$ intervalles égaux et posons

$$
\theta_{\imath}=\frac{\pi}{2 n} . i, \quad i=0,1, \ldots, n
$$

On vérifie aisément que si $n \geqq \pi /[4 \operatorname{Arc} \cos (1 /(1+\varepsilon))]$ nous pouvons poser

$$
\mathscr{U}_{\varepsilon}^{+}(2)=\left\{\left(\begin{array}{c}
\cos \theta_{\imath} \\
\sin \theta_{\imath}
\end{array}\right), i=0,1, \ldots, n\right\} .
$$

Construction récurrente d'ensembles $\mathscr{U}_{\varepsilon}(p)$ et $\mathscr{U}_{\varepsilon}^{s}(p)$. Soit

$$
p=p_{1}+p_{2}, \quad p_{1} \in \mathbf{N}^{*}, \quad p_{2} \in \mathbf{N}^{*}
$$

Nous pouvons écrire tout vecteur $u$ de $\mathscr{U}(p)$ sous la forme

$$
u=\left(\begin{array}{cc}
a^{1} u^{1} \\
a^{2} u^{2}
\end{array}\right), \quad u^{1} \in \mathscr{U}\left(p_{1}\right), \quad u^{2} \in \mathscr{U}\left(p_{2}\right), \quad\left(\begin{array}{c}
a^{1} \\
a^{2}
\end{array}\right) \in \mathscr{U}^{+}(2) .
$$

Propriété : Soient $\varepsilon$ et $\eta$ deux réels positıfs et des ensembles

$$
\mathscr{U}_{\eta}\left(p_{1}\right) \mathscr{U}_{\eta}\left(p_{2}\right) \mathscr{U}_{\eta}^{s}\left(p_{1}\right) \mathscr{U}_{\eta}^{+}(2)
$$

Si $\eta \leqq \sqrt{1+\varepsilon}-1$ nous pouvons poser

et

$$
\mathscr{U}_{\varepsilon}(p)=\left\{\left(\begin{array}{c}
\alpha^{1} v^{1} \\
\alpha^{2} v^{2}
\end{array}\right): v^{1} \in \mathscr{U}_{\eta}\left(p_{1}\right), v^{2} \in \mathscr{U}_{\eta}\left(p_{2}\right),\left(\begin{array}{c}
\alpha^{1} \\
\alpha^{2}
\end{array}\right) \in \mathscr{U}_{\eta}^{+}(2)\right\}
$$

$$
\mathscr{U}_{\varepsilon}^{s}(p)=\left\{\left(\begin{array}{c}
\alpha^{1} v^{1} \\
\alpha^{2} v^{2}
\end{array}\right): v^{1} \in \mathscr{U}_{\eta}^{s}\left(p_{1}\right), v^{2} \in \mathscr{U}_{\eta}\left(p_{2}\right),\left(\begin{array}{c}
\alpha^{1} \\
\alpha^{2}
\end{array}\right) \in \mathscr{U}_{\eta}^{+}(2)\right\}
$$


Démonstration Nous ferons la démonstration pour $\mathscr{U}_{\varepsilon}(p)$ la transposition pour $\mathscr{U}_{\varepsilon}^{s}(p)$ étant triviale

Solt $u \in \mathscr{U}(p)$ et écrivons

$$
u=\left(\begin{array}{cc}
a^{1} & u^{1} \\
a^{2} u^{2}
\end{array}\right), \quad u^{1} \in \mathscr{U}\left(p_{1}\right), \quad u^{2} \in \mathscr{U}\left(p_{2}\right), \quad\left(\begin{array}{c}
a^{1} \\
a^{2}
\end{array}\right) \in \mathscr{U}^{+}(2),
$$

choisissons $v^{1} \in \mathscr{U}_{\eta}\left(p_{1}\right) v^{2} \in \mathscr{U}_{\eta}\left(p_{2}\right)$ et $\left(\begin{array}{c}\alpha^{1} \\ \alpha^{2}\end{array}\right) \in \mathscr{U}_{\eta}^{+}(2)$ tels que

$$
a^{1} \alpha^{1}+a^{2} \alpha^{2} \geqq \frac{1}{1+\eta}, \quad u^{1 T} v^{1} \geqq \frac{1}{1+\eta}, \quad u^{2 T} v^{2} \geqq \frac{1}{1+\eta}
$$

Nous avons

$$
u^{T}\left(\begin{array}{c}
\alpha^{1} v^{1} \\
\alpha^{2} v^{2}
\end{array}\right)=a^{1} \alpha^{1} u^{1 T} v^{1}+a^{2} \alpha^{2} u^{2 T} v^{2}
$$

donc

$$
u^{T}\left(\begin{array}{c}
\alpha^{1} v^{1} \\
\alpha^{2} v^{2}
\end{array}\right) \geqq \frac{a^{1} \alpha^{1}}{1+\eta}+\frac{a^{2} \alpha^{2}}{1+\eta} \geqq \frac{1}{(1+\eta)^{2}} \geqq \frac{1}{1+\varepsilon}
$$

Corollaire Construction d'ensembles $\mathscr{U}_{\varepsilon}(p)$ et $\mathscr{U}_{\varepsilon}^{s}(p)$ finis

Pour tout $\varepsilon>0$ et tout $p \in \mathbf{N}^{*}$ nous pouvons construire des ensembles $\mathscr{U}_{\varepsilon}(p)$ et $\mathscr{U}_{\varepsilon}^{s}(p)$ finis

Démonstration Pour $p \leqq 2$ nous avons déjà montré au début du paragraphe 24 comment construire des ensembles $\mathscr{U}_{\varepsilon}(p), \mathscr{U}_{\varepsilon}^{s}(p)$ et $\mathscr{U}_{\varepsilon}^{+}(p)$ finis

Supposons donc que nous puissions construire pour $p \leqq \bar{p}$ des ensembles $\mathscr{U}_{\varepsilon}(p) \mathscr{U}_{\varepsilon}^{s}(p)$ finis pour tout $\varepsilon>0$ si $\bar{p}+1=2 q, q \in \mathbf{N}^{*}$ posons $p_{1}=p_{2}=q$ et s1 $\bar{p}+1=2 q+1, q \in \mathbf{N}^{*}$ posons $p_{1}=q+1, p_{2}=q$

Le résultat de la propriété précédente montre que l'on peut construire $\mathscr{U}_{\varepsilon}(\bar{p}+1)$ de la manière suivante

$$
\mathscr{U}_{\varepsilon}(\bar{p}+1)=\left\{\left(\begin{array}{c}
\alpha^{1} v^{1} \\
\alpha^{2} v^{2}
\end{array}\right), v^{1} \in \mathscr{U}_{\eta}\left(p_{1}\right), v^{2} \in \mathscr{U}_{\eta}\left(p_{2}\right),\left(\begin{array}{c}
\alpha^{1} \\
\alpha^{2}
\end{array}\right) \in \mathscr{U}_{\eta}^{+}(2)\right\}
$$

avec $\eta=\sqrt{1+\varepsilon}-1$ Si $\mathscr{U}_{\eta}\left(p_{1}\right), \mathscr{U}_{\eta}\left(p_{2}\right)$ et $\mathscr{U}_{\eta}^{+}(2)$ sont finis $\mathscr{U}_{\varepsilon}(\bar{p}+1)$ est alors finı, or $p_{1} \leqq \bar{p}, p_{2} \leqq \bar{p}$ et $2 \leqq \bar{p}$

Nous pouvons de même construire $\mathscr{U}_{\varepsilon}^{s}(\bar{p}+1)$ finı en posant

$$
\mathscr{U}_{\varepsilon}^{s}(\bar{p}+1)=\left\{\left(\begin{array}{c}
\alpha^{1} v^{1} \\
\alpha^{2} v^{2}
\end{array}\right), v^{1} \in \mathscr{U}_{\eta}^{s}\left(p_{1}\right), v^{2} \in \mathscr{U}_{\eta}\left(p_{2}\right),\left(\begin{array}{c}
\alpha^{1} \\
\alpha^{2}
\end{array}\right) \in \mathscr{U}_{\eta}^{+}(2)\right\}
$$

Cec1 montre donc que nous pouvons construire $\mathscr{U}_{\varepsilon}(p)$ et $\mathscr{U}_{\varepsilon}^{s}(p)$ finis pour tout $\varepsilon>0$ et $p \in \mathbf{N}^{*}$, le procédé de récurrence pouvant être utılısé de manière 
constructive pour exprimer un ensemble $\mathscr{U}_{\varepsilon}(p)$ ou $\mathscr{U}_{\varepsilon}^{s}(p)$ uniquement en fonction d'un certain nombre fin1 d'ensembles $\mathscr{U}_{\varepsilon}\left(p_{\imath}\right), p_{\imath} \leqq 2$

Nombre d'élements de $\mathscr{U}_{\varepsilon}(p)$ et $\mathscr{U}_{\varepsilon}^{s}(p)$ Des formules de récurrence donnant le nombre d'élćments des ensembles construits de la maniere précedente peuvent être établies [3] Nous donnons 1c1 seulement des tableaux comportant quelques valeurs de ce nombre d'éléments

\begin{tabular}{|l|r|r|r|}
\hline \multicolumn{5}{|c|}{ Nombres d'elements de $\mathscr{U}_{\varepsilon}(p)$} \\
\hline \multicolumn{1}{|c|}{$\varepsilon$} & 005 & 01 & 015 \\
\hline 3 & 11 & 8 & 7 \\
\hline 4 & 107 & 57 & 47 \\
\hline 5 & 705 & 264 & 180 \\
\hline
\end{tabular}

\begin{tabular}{|c|c|c|c|}
\hline \multicolumn{4}{|c|}{ Nombres d elements de $\mathscr{U}_{\varepsilon}^{s}(p)$} \\
\hline$\varepsilon$ & 005 & 01 & 015 \\
\hline 2 & 6 & 4 & 4 \\
\hline 3 & 58 & 32 & 27 \\
\hline 4 & 383 & 149 & 104 \\
\hline 5 & 5673 & 1345 & 617 \\
\hline
\end{tabular}

\subsection{Application à l'exemple introductif}

Nous donnons $1 \mathrm{c1}$ quelques temps moyens de calcul (sur IBM 360-67) pour résoudre le probleme de l'exemple introductif avec une matrice de talle $2 n=100$ pour differentes valeurs de $p$ et de la precision $\varepsilon$ Rappelons ( $c f$ \& 1 2) qu'il s'agit d'un problème symétrique et que nous utılisons donc $\mathscr{U}_{\varepsilon}^{s}(p)$

\begin{tabular}{|l|c|c|c|}
\hline$p$ & 005 & 01 & 015 \\
\hline 2 & 03 & 024 & 022 \\
\hline 4 & 104 & 41 & 3 \\
\hline
\end{tabular}

Les temps sont donnes en secondes

\section{EXEMPLES. GENERALISATIONS DE L'EXEMPLE INTRODUCTIF ET PROBLEMES} COMBINATOIRES SUR UN ENSEMBLE DE POINTS DE $\mathbf{R}^{p}$

Après avoir sıgnalé brièvement quelques géneralısations de l'exemple introductıf nous donnons dans ce chapitre deux problèmes combinatoires n'ayant pas a prior de solution triviale, leur formulation en terme de probleme 
de distance euclidienne maximale, en explicitant la resolution des problèmes projetés qui, elle, est aısée cecı permet donc d'appliquer la méthode décrite au paragraphe 2 Ces problèmes concernent la recherche de "formes » dans des ensembles de points de $\mathbf{R}^{p}$ et 1 l semble que, même en dehors des exemples donnés c1-dessous, l'ut1lisation des problèmes projetés soit assez fondamentale pour ce type de problèmes (recherche d'enveloppe convexe )

\subsection{Généralisation de l'exemple introductif}

Nous prenons maintenant une matrice A de $\mathcal{M}_{n, n}(\mathbf{R})$ (la dimensıon n'étant pas obligatorrement parre) toujours donnée par

$$
A=\sum_{\imath=1}^{p} B_{\imath} B_{\imath}^{T} \quad \text { ou } \quad B_{\imath} \in \mathbf{R}^{n}, l=1,2, \quad, p
$$

et $p$ est de l'ordre de 5

Solt $E=\{1,2, \quad, n\}$ Nous désignerons par $E_{1}$ et $E_{2}$ deux sous-ensembles constituant une partition de $E$ et nous noterons $n_{1}=\operatorname{Card}\left(E_{1}\right), n_{2}=\operatorname{Card}\left(E_{2}\right)$

Les problèmes suivants sont susceptibles d'une méthode de résolution identıque à celle de l'exemple introductıf

$n_{1}$ et $n_{2}$ étant fixés $\left(n_{1}+n_{2}=n\right)$ trouver $E_{1}\left(\right.$ et $\left.E_{2}\right)$ tels que $\sum_{\substack{i \in E_{1} \\ j \in E_{2}}} A(l, j)$ soit

$\underline{n} \bar{n}$ étant fixes trouver $E_{1}\left(\right.$ et $\left.E_{2}\right)$ tels que $\underline{n} \leqq n_{1} \leqq \bar{n}$ et $\sum_{\substack{l \in E_{1} \\ j \in E_{2}}} A(l, j)$ solt minımum

$\underline{n} \bar{n}$ étant fixés trouver $E_{1}\left(\right.$ et $\left.E_{2}\right)$ tels que $\underline{n} \leqq n_{1} \leqq \bar{n}$ et $\sum_{\substack{i \in E_{1} \\ j \in E_{1}}} A(l, J)$ solt maxımum

\subsection{Notations}

Solt un ensemble de $n$ points de $\mathbf{R}^{p} \quad\left\{X_{1}, X_{2}, \quad, X_{n}\right\}$ et $B_{1}, B_{2}, \quad, B_{p}$ les vecteurs de $\mathbf{R}^{n}$ définıs par

$$
B_{J}(l)=X_{\imath}(J), \quad l=1,2, \quad, n, \quad J=1,2, \quad, p
$$

Afin de simplifier certaines expressions nous associerons à une partition des $n$ points en deux groupes 1 et 2 , un couple de vecteurs de $\mathbf{R}^{n},\left(V_{1}, V_{2}\right)$ vérifiant

$$
\left\{\begin{array}{c}
V_{k}(l)=\left\{\begin{array}{ll}
0, & k=1,2, \\
1, & l=1,2,
\end{array}, n\right. \\
V_{1}(l)+V_{2}(l)=1, \quad l=1,2, \quad, n,
\end{array}\right.
$$

$V_{k}(l)=1$ signifiant que $X_{1}$ appartient au groupe $k$ 
Nous poserons

$$
d_{1}=\sum_{i=1}^{n} V_{1}(i), \quad d_{2}=\sum_{i=1}^{n} V_{2}(i)
$$

\subsection{Séparation optimale des centres de gravité}

Énoncé du problème : Soit $G_{1}$ le centre de gravité du premier groupe de point $G_{2}$ celui du second. On cherche la partition de l'ensemble des $n$ points qui maximise la distance euclidienne usuelle de $G_{1}$ à $G_{2}$.

Énoncé en terme de problème de "distance euclidienne maximale » : Avec les notations 3.2 la distance euclidienne usuelle de $G_{1}$ à $G_{2}$ est

$$
\left(\sum_{J=1}^{p}\left(\frac{V_{1}^{T} B_{J}}{d_{1}}-\frac{V_{2}^{T} B_{J}}{d_{2}}\right)^{2}\right)^{1 / 2}
$$

Le problème posé est donc de maxımiser la norme $\varphi_{2}$ sur l'ensemble

$$
\mathscr{D}=\left\{X \in \mathbf{R}^{p}: X(j)=\frac{V_{1}^{T} B_{j}}{d_{1}}-\frac{V_{2}^{T} B_{J}}{d_{2}}\left(V_{1}, V_{2}\right) \text { vérifiant } \mathrm{C}\right\} .
$$

La méthode de résolution utilisant les problèmes projetés nous donnera pour un point $X$ proposé le couple $\left(V_{1}, V_{2}\right)$ correspondant donc effectivement la partition.

«Problèmes projetés » : Soit $u \in \mathscr{U}(p)$ et

$$
K=V_{1}-V_{2}, \quad V=V_{1}+V_{2}=\left(\begin{array}{c}
1 \\
1 \\
\vdots \\
1
\end{array}\right)
$$

Pour $X \in \mathscr{D}$ associé à $\left(V_{1}, V_{2}\right)$ nous avons

$$
X^{t} u=\frac{n}{2 d_{1} d_{2}} K^{T}\left(\sum_{J=1}^{p} u(j) B_{J}\right)+\frac{d_{2}-d_{1}}{2 d_{1} d_{2}} V^{T}\left(\sum_{J=1}^{p} u(j) B_{J}\right) .
$$

Si $d_{1}$ et $d_{2}$ sont fixés le problème projeté se résoud en maximisant la quantité $K^{T}\left(\sum_{l=1}^{p} u(j) B_{J}\right)$ parmi les vecteurs $K$ comportant $d_{1}$ composantes +1 et $d_{2}$ composantes -1 . Un résultat analogue à celui du lemme du paragraphe 1.3 montre que ce problème est résolu lorsque les composantes du vecteur $\sum_{j=1}^{p} u(j) B_{J}$ ont été ordonnées. Le tri étant le même pour chaque valeur de $d_{1}$ 
et $d_{2}$ nous en déduisons la solution du problème $\operatorname{Max} X^{T} u$ par simple comparaison ces valeurs obtenues pour chaque couple $\left(d_{1}, d_{2}\right)$.

\subsection{Minimisation d'un critère d'écart}

Énoncé du problème: Nous nous proposons de trouver la partition de l'ensemble des $n$ points qui minimise

$$
\sum_{X_{\imath} \in \text { groupe } 1} \varphi_{2}^{2}\left(X_{\imath}-G_{1}\right)+\sum_{X_{i} \in \text { groupe } 2} \varphi_{2}^{2}\left(X_{\imath}-G_{2}\right) .
$$

Énoncé en terme de problème de « distance euclidienne maximale »: Le problème de minimisation précédent est équivalent au problème de maximisation de la norme euclidienne $\varphi^{2}$ sur :

$$
\begin{aligned}
\mathscr{D}=\left\{X \in \mathbf{R}^{p}: X(j)=\frac{K^{T} B_{j}}{\sqrt{d_{2} d_{1}}}+V^{T} B_{J} \frac{d_{2}-d_{1}}{\sqrt{d_{2} d_{1}}},\right. \\
\left.K=V_{1}-V_{2},\left(V_{1}, V_{2} \text { vérifiant C }\right)\right\} .
\end{aligned}
$$

Démonstration: Introduisons deux matrices diagonales de $M_{n, n}(\mathbf{R}) D_{1}$ et $D_{2}$ telles que :

$$
D_{1}(i, i)=V_{1}(i), \quad D_{2}(i, i)=V_{2}(i), \quad i=1,2, \ldots, n .
$$

Nous avons

$$
\sum_{X_{1} \in \text { groupe } 1} \varphi_{2}^{2}\left(X_{\imath}-G_{1}\right)=\sum_{X_{\imath} \in \text { groupe } 1} \sum_{J=1}^{p}\left(X_{\imath}(j)-\frac{V_{1}^{T} B_{J}}{d_{1}}\right)^{2},
$$

ce qui est égal à

$$
\sum_{j=1}^{p}\left(D_{1} B_{j}-\frac{V_{1}^{T} B_{J}}{d_{1}} V_{1}\right)^{T}\left(D_{1} B_{J}-\frac{V_{1}^{T} B_{j}}{d_{1}} V_{1}\right)
$$

et donc à

$$
\sum_{j=1}^{p}\left(B_{J}^{T} D_{1} B_{J}-\frac{\left(V_{1}^{T} B_{j}\right)^{2}}{d_{1}}\right)
$$

Le critère à minimiser est en fin de compte

$$
\sum_{J=1}^{p} B_{J}^{T} B_{J}-\sum_{J=1}^{p}\left\{\frac{\left(V_{1}^{T} B_{J}\right)^{2}}{d_{1}}+\frac{\left(V_{2}^{T} B_{j}\right)^{2}}{d_{2}}\right\}
$$

vol $13, \mathrm{n}^{\circ} 2,1979$ 
En introduisant le vecteur $K=V_{1}-V_{2}$ la quantité précédente est égale à

$$
\sum_{J=1}^{p}\left(B_{J}^{T} B_{J}-\frac{\left(V^{T} B_{J}\right)^{2}}{n}\right)-\frac{n}{4} \sum_{J=1}^{p}\left(\frac{K^{T} B_{J}}{\sqrt{d_{1} d_{2}}}+\frac{d_{2}-d_{1}}{n \sqrt{d_{1} d_{2}}} V^{T} B_{J}\right)^{2} .
$$

Le premier terme étant constant nous sommes donc ramenés à la maximisation de

$$
\sum_{j=1}^{p}\left(\frac{K^{T} B_{J}}{\sqrt{d_{1} d_{2}}}+\frac{d_{2}-d_{1}}{n \sqrt{d_{1} d_{2}}} V^{T} B_{J}\right)^{2}
$$

"Problème projeté »: Pour $X \in \mathscr{D}$ associé à $K$ nous avons

$$
X^{T} u=K^{T} \sum_{j=1}^{p} \frac{u(j) B_{J}}{\sqrt{d_{1} d_{2}}}+\sum_{j=1}^{p} \frac{u(j)\left(d_{2}-d_{1}\right)}{n \sqrt{d_{1} d_{2}}} V^{T} B_{J}
$$

Les problèmes de maximisation à $d_{1}$ et $d_{2}$ fixés se résolvent $(c f . \S 1.3)$ à l'aide d'un tri du vecteur $\sum_{j=1}^{p} u(j) B_{J}$. Ce tri étant le même pour chaque valeur de $d_{1}, d_{2}$ on en déduit aisément la solution du problème projeté.

\section{CONCLUSION. UTILISATION PRATIQUE DES ALGORITHMES ÉNUMÉRATIFS}

Notons d'abord que dans les exemples que nous avons traités, les résultats obtenus sont très fréquemment meilleurs que le laisseraient prévoir les précisions demandées. Ces corrections peut même être rigoureusement évaluées a posteriori [3].

Signalons aussi que l'utilisation à de faibles précisions des algorithmes énumératifs fournit un moyen de choisir les points de départ d'algorithmes locaux d'optimisation.

\section{MODIFICATION ET VARIANTES DES ALGORITHMES ENUMÉRATIFS}

Dans le cas $p=2$ nous avons pu, toujours par l'utilisation des problemes projetés, mettre au point des algorithmes non énumératifs et permettant d'atteindre les précisions habituellement demandées [3].

Remarquons aussi que des variantes des algorithmes décrits au paragraphe 2 peuvent être développées à partır de résolutions approchées des problèmes projetés. De même les ensembles $\varkappa_{\varepsilon}(p)$ pourraient être remplacés par des ensembles $\mathscr{U}_{\varepsilon}^{\mathscr{Q}}(p)$ comportant moins d'éléments mais construits avec certaines informations sur l'ensemble $\mathscr{D}$ (localisation du maximum par exemple). 


\section{PERSPECTIVES}

La méthode proposée n'a pas pour ambition d'être universelle pour tous les problèmes $\operatorname{Max} \varphi_{2}(X)$

Notons sımplement qu'elle nous a permıs d'obtenır des résultats satısfaisants pour des problèmes pratıquement insolubles par les méthodes usuelles d'optimisation Sur la vue des quelques principes dont nous avons parlé en conclusion 1 nous semble raisonnable de penser que cette méthode puisse servir de base à quelques algorithmes de résolution de problèmes voisıns

\section{BIBLIOGRAPHIE}

1 L C W Dixon et G P Szego Towards Global Optımization Proceedings of workshop at the Unıversity of Caglıarı Italie octobre 1974

2 R Karp On the Computational Complexity of Combinatorial Problems, Networks, 5 , 1975

3 B LACOLLE Quelques problemes de restructuration dans un environnement pagine These de $3^{e}$ cycle, Mathematiques, Université de Grenoble, 1976

4 B LACOLLE, Resolution approchee d'un probleme combinatoure de partitionnement par une enumeration partielle, Colloque d'Analyse numerique Imbours, 1977 